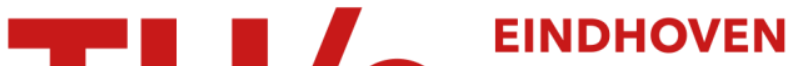 UNIVERSITY OF TECHNOLOGY
}

\section{Maintenance optimization under non-constant probabilities of imperfect inspections}

\section{Citation for published version (APA):}

Driessen, J. P. C., Peng, H., \& van Houtum, G. J. J. A. N. (2017). Maintenance optimization under non-constant probabilities of imperfect inspections. Reliability Engineering and System Safety, 165(1), 115-123.

https://doi.org/10.1016/j.ress.2017.03.020

DOI:

10.1016/j.ress.2017.03.020

Document status and date:

Published: 01/09/2017

\section{Document Version:}

Accepted manuscript including changes made at the peer-review stage

\section{Please check the document version of this publication:}

- A submitted manuscript is the version of the article upon submission and before peer-review. There can be important differences between the submitted version and the official published version of record. People interested in the research are advised to contact the author for the final version of the publication, or visit the $\mathrm{DOI}$ to the publisher's website.

- The final author version and the galley proof are versions of the publication after peer review.

- The final published version features the final layout of the paper including the volume, issue and page numbers.

Link to publication

\section{General rights}

Copyright and moral rights for the publications made accessible in the public portal are retained by the authors and/or other copyright owners and it is a condition of accessing publications that users recognise and abide by the legal requirements associated with these rights.

- Users may download and print one copy of any publication from the public portal for the purpose of private study or research.

- You may not further distribute the material or use it for any profit-making activity or commercial gain

- You may freely distribute the URL identifying the publication in the public portal.

If the publication is distributed under the terms of Article 25fa of the Dutch Copyright Act, indicated by the "Taverne" license above, please follow below link for the End User Agreement:

www.tue.nl/taverne

Take down policy

If you believe that this document breaches copyright please contact us at:

openaccess@tue.nl

providing details and we will investigate your claim. 


\title{
Maintenance optimization under non-constant probabilities of imperfect inspections
}

\author{
J.P.C. Driessen ${ }^{a}$, H. Peng ${ }^{b}$, G.J. van Houtum ${ }^{a *}$ \\ ${ }^{a}$ Eindhoven University of Technology, School of Industrial Engineering, the Netherlands \\ ${ }^{b}$ Academy of Mathematics and Systems Science, Chinese Academy of Sciences, China
}

\begin{abstract}
In this research, we study a single-component system that is characterized by three distinct deterioration states, cf. the Delay Time Model: normal, defective, and failed. The system is inspected periodically, and preventive system maintenance after a given number of inspections. The inspections are imperfect, and the probability of an inspection error changes over the system's operation time. Our objective is to minimize the average cost over an infinite time horizon. We present exact cost evaluations for a given maintenance policy, and we compare our model with non-constant probabilities to a model that considers constant probabilities of inspection errors. Our computational study illustrates that the model with constant probabilities may yield, on average, $19 \%$ higher costs than the model using non-constant probabilities of inspection errors. These values depend on the chosen parameter values, but still give an indication of how large the difference between both models can be. Finally, we also present an extension in which a reliability constraint (in terms of average failures per time unit) is added to our problem.
\end{abstract}

Keywords: Maintenance Optimization, Delay-Time Model, False Positives, False Negatives, Human Factors

\section{Introduction}

Unexpected failures cause costly downtime for many advanced technical systems, such as airplanes, trains, baggage handling systems, and medical systems. Maintenance is done during system operation to avoid such unexpected failures. The costs of these maintenance activities comprise 15-60\%

${ }^{*}$ Corresponding author, Tel.: +31 40247 5163, E-mail: g.j.v.houtum@tue.nl 
of the total production costs in a manufacturer's facility (Mobley, 2002). In such situations, it is important to minimize the maintenance costs. Mathematical maintenance models and techniques are typically used to support this objective by deriving optimal maintenance policies.

The literature on maintenance optimization is rich and covers various areas such as system replacement, inspections, repair, and maintenance scheduling (Jardine and Tsang, 2013). These areas of maintenance optimization use modeling techniques that describe system degradation. There exists a large variety of models describing system degradation differently, e.g. continuous degradation or discrete state degradation. Many of these models have in common that they also consider inspections that reveal the system's level of degradation. For instance, Whitmore (1995), and Newby and Dagg (2004) model the continuous degradation with by a Wiener process and assume imperfect inspections that may contain noise. In other work, Kallen and van Noortwijk (2005) propose to model system degradation by a Gamma process, and they also assume inspections with noise. On the other hand, system degradation is also modeled in terms of discrete states, contrary to continuous degradation. Some researchers have proposed multi-state Markovian degradation models that include imperfect inspections, see for example Welte (2009), and Le and Tan (2013).

Another common approach to modeling system degradation in terms of discrete states is the Delay Time Model (DTM). This model distinguishes three deterioration states: normal, defective, and failed. The system operates properly in the normal state; operates in the defective state as well, but its defect can be revealed by inspections; or the system has failed. The time the system spends in the normal and defective state are called the time to defect and the delay time, respectively. Analogous to other maintenance models in literature, the DTM is typically studied under inspection based maintenance policies; i.e., inspections are done to reveal the system's degradation. A literature overview of the DTM up to 1999 is provided by Christer (1999), and Wang (2012) reviews the research from 1999 to 2012 . The most recent advancements, since 2012, include postponements of maintenance actions when the defects are detected (van Oosterom et al., 2014), and the combination of multiple different forms of preventive maintenance activities - such as routine service, preventive system maintenance, and inspections - based on the DTM (Wang, 2013).

The DTM literature considering inspection policies generally assumes that the inspections are perfect. However, inspections are usually not perfect in practice due to errors, such as human errors or errors of measurement equipment (Wickens, 1992). Therefore, imperfect inspections have been included in single-component DTMs by Okumura et al. (1996) and Berrade et al. (2013). Both works consider two types of imperfect inspection behavior, i.e., false positives and false negatives. 
A false positive corresponds to the judgment that a system is in its defective state, when it is actually in its normal state. False negatives correspond to the judgment that a system is in its normal state when, in fact, the system is in its defective state. We refer to the probabilities of false positives and false negatives by $\alpha$ and $\beta$, respectively. For an overview, Table 1 is included.

Table 1: Probabilities of inspection behavior

\begin{tabular}{lccc}
\hline & & \multicolumn{2}{c}{ System State } \\
\cline { 3 - 4 } & & Normal & Defective \\
Inspection outcome & Normal & $1-\alpha$ & $\beta$ \\
& Defective & $\alpha$ & $1-\beta$ \\
\hline
\end{tabular}

The probabilities of false positives and false negatives, $\alpha$ and $\beta$, are assumed to be constant over time by Okumura et al. (1996) and Berrade et al. (2013). However, this approach might be inaccurate. Maintenance engineers may become more tempted to engage in a false positive when the system has been operating (in its normal state) for a longer period of time. That is, they use their own judgement of the time the system has been operating normally instead of the actual inspection outcome. Such behavior implies that the probability of false positives is non-constant, and depends on the time the system has been operating in the normal state. This approach to false positives has not been explored in the DTM literature, nor in any of the other maintenance models. Therefore, our work extends the literature by proposing a non-constant probability of false positives for a single-component DTM. Furthermore, maintenance engineers may encounter difficulties in determining whether a system has become defective at early stages of system degradation, e.g. smaller cracks are more difficult to find than larger ones (Forsyth and Fahr, 1998). Therefore, the probability of false negatives is non-constant and depends on the system degradation. We extend the literature by relating this probability of false negatives to the system's duration in the defective state relative to its delay time, which is a measure of system degradation. By this way of modeling the probability of false negatives, we provide a richer concept of system degradation compared to Wang (2010), who conceptualizes system degradation by only considering the duration that a system has been defective. Let us illustrate the enhanced richness of our concept by means of an example. Weaker materials typically have a lower delay time compared to stronger materials. Hence, under the same duration in the defective state, the weaker material (shorter delay time) will have a higher level of degradation than the strong one. Table 2 presents a schematic literature 
overview of DTMs with imperfect inspections.

Table 2: Imperfect inspections in the DTM literature

\begin{tabular}{|c|c|c|c|}
\hline \multirow{4}{*}{ False Negatives } & & \multicolumn{2}{|c|}{ False Positives } \\
\hline & & Constant & Non-constant \\
\hline & Constant & $\begin{array}{c}\text { Okumura et al. (1996); } \\
\text { Berrade et al. (2013) }\end{array}$ & \\
\hline & Non-constant & Wang (2010) & This research \\
\hline
\end{tabular}

In this research, we propose a single-component model that considers non-constant probabilities of false positives and false negatives, and we will refer to this model as the true model. Next to the true model, we also study the approximate model, which is a single-component model that considers constant probabilities of false positives and false negatives. This approximate model is typically easier to use and to implement due to the constant probabilities. Furthermore, the approximate model will serve as a comparison to the true model in this paper. This enables us to shed some light on the benefit of modeling non-constant probabilities of imperfect inspections over modeling the probabilities as constants. Our objective is to minimize the average cost per time unit over an infinite time horizon by optimizing the maintenance policy. Our research's contributions are twofold: (1) we present an exact cost evaluation of our true model; and (2) we compare our true model to the approximate model. We show that the approximate model may result in policies that yield - on average - 19\% higher cost than the true model. This implies that the reduction in model complexity comes at an average cost increase of $19 \%$, based on our instances tested. We would like to emphasize that these numbers depend on the parameter settings, but they still give an indication of the extent that the two models may differ.

The remainder of this paper is organized as follows. In Section 2, we present the model. In Section 3, we give an exact cost evaluation for our maintenance policy, and we discuss the optimization procedure. In Section 4, we present a method for comparing the true and approximate model, and we present the computational results that compare both models in Section 5 . We present an extension including a reliability constraint in Section 6, and we conclude our work in Section 7. 


\section{Model description}

In this section, we describe our true model. However, the description and the reasoning also applies to the approximate model. The sole difference is that, in the latter case, the probabilities of false positives and false negatives are assumed to be constants.

Let us consider a single-component system operating over an infinite time horizon. The system has three states: normal, defective and failed. In the normal operating state, the system is working properly, without any detectable defects. In the defective state, inspections may reveal the system's defect. Yet, the system is still able to operate. The failed state of the system is self-announcing, and the system stops delivering its function immediately. If the system fails, it is replaced correctively. To prevent the system from reaching its failed state, it is inspected periodically each $T>0$ time units, it is preventively replaced upon detection of the defective state, or it is preventively replaced after $M-1$ inspections at time $M T$. This implies that at time $M T$ we do not perform another inspection, as the system is preventively replaced independent on its state, i.e., the $M^{\text {th }}$ inspection is unnecessary. For more details on such a policy, see Scarf et al. (2009). We denote our maintenance policy by $(M, T)$, and note that it degenerates to an age-based maintenance policy when $M=1$, it reduces to a pure inspection policy when $M=\infty$, and it is a hybrid policy for any finite $M>1$. We assume that inspections are the only means to detect the normal and defective state.

We denote the duration of the time that the system is in the normal state, referred to as the time to defect, by the continuous random variable $X>0$. This time to defect corresponds to the time between maintenance (preventive or corrective), and the arrival time of the defect. The random time the system takes from defect arrival to failure, without doing any maintenance, is referred to as the delay time and denoted by the continuous random variable $H>0$. The sum of both random variables is the system's time to failure. The cumulative distribution function (cdf) and the probability density function (pdf), corresponding to both state durations, are defined by $F_{X}(\cdot)$ and $f_{X}(\cdot)$ for the time to defect, and by $F_{H}(\cdot)$ and $f_{H}(\cdot)$ for the delay time, respectively.

The cost for performing an inspection is denoted by $c_{i}$, and the cost $c_{p}$ is incurred for preventive maintenance. When the system unexpectedly fails in between inspections, corrective maintenance is immediately carried out with $\operatorname{cost} c_{c}$. We assume that the inspection schedule restarts after system failure; i.e., the first inspection is performed $T$ time units after system failure, see also Wang (2010) and Berrade et al. (2013). We assume that the failure cost, containing for example collateral damage cost, is included in the corrective maintenance cost $c_{c}$. Furthermore, we assume 
that $0<c_{i}<c_{p}<c_{i}+c_{p}<c_{c}$, and that the time for inspections and maintenance is negligible.

In our model, the inspections can be imperfect; i.e., an inspection error can occur, and the inspection outcome differs from the system's true state. We take two classes of imperfect inspections into account: (1) false positives; and (2) false negatives. For an overview, see Table 1. The probabilities of imperfect inspections are assumed to be non-constant. Hence, we use two functions $\alpha(\cdot)$ and $\beta(\cdot)$ to describe the non-constant probabilities of false positives and false negatives, respectively.

For the non-constant probability of false positives, we assume that some engineers do not use the measurement outcome in their judgment, but replace this with a substitute attribute (Kahneman, 2003). Our rationale behind this substitute attribute is that engineers become more tempted to preventively replace a system too early, when it has been working properly for a longer time. Thus, we consider the time $t$ that the system has been operating in its normal state as the substitute attribute. This time starts from the latest maintenance moment (preventive or corrective). We assume that, if the time $t$ of an inspection approaches a threshold value $a$, the engineers (using the time $t$ as a substitute attribute) will become more tempted to engage in a false positive. Moreover, we assume - for simplicity - that all maintenance engineers that use the substitute attribute consider the same threshold $a$. However, our analysis (Section 3) and comparison method (Section 4) also apply when each engineer uses an individual piecewise linear function. In this case, the variable $a$ becomes a random variable. The variable $a$ might relate to a temporal parameter at which the maintenance engineers believe the system typically becomes defective, e.g. the mean time to defect. If the time $t$ of an inspection exceeds the threshold value $a$, we assume that the engineers using the attribute substitution, will preventively replace the system. The probability of $\alpha(t)$ is then increasing for $t<a$, and remains constant for $t \geq a$. Note that $\alpha(a)<1$ when not all maintenance engineers use the substitute attribute and $\alpha(a)=1$ when all maintenance engineers do. Furthermore, we do not make any further restrictions on the functional form of $\alpha(t)$, and Figure 1 shows an example of a simple piecewise function for $\alpha(t)$.

When the defect appears at the realization $x$ of the time to defect, we assume that the system starts its degradation until failure. Such system degradation has been extensively investigated in terms of the Probability Of Detection (POD). This POD is defined as the probability of finding a degradation when it is present (Berens, 1989). It reflects the probability of false negatives by $\beta=1-P O D$ (Nichols et al., 2008). As a system degrades, the POD increases; i.e., the probability of false negatives decreases with system degradation (Forsyth and Fahr, 1998). We conceptualize this 
system degradation by the failure progress. If the realized delay time is $h$; i.e., the failure occurs at $h$ time units after the defect arrival, the failure progress is defined by the relative duration $(t-x) / h$ in the defective state, for $x \leq t \leq \min \{x+h, M T\}$. Similar to the POD literature, we assume that the inspection engineers have difficulty in determining whether degradation exists at the early stage of the failure progress. The more the system degrades, the easier the detection of the degradation becomes, e.g. larger cracks are easier to detect smaller ones (Forsyth and Fahr, 1998). Hence, the probability of false negatives $\beta((t-x) / h)$ is nonincreasing in $(t-x) / h$. We refer to Berens (1989) for a more detailed discussion. An illustration for $\beta((t-x) / h)$ that is consistent with the POD literature is presented in Figure 1. We would like to emphasize that we do not make any restrictions on the functional form of $\beta((t-x / h))$, and Figure 1 illustrates a function as described in the POD literature.
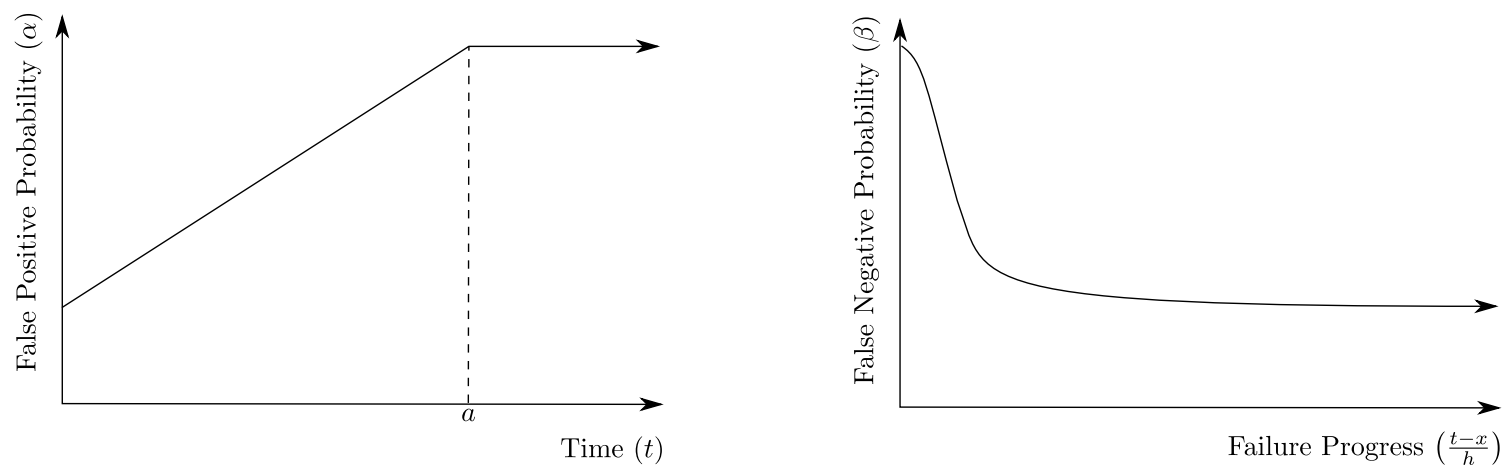

Figure 1: Illustration of non-constant probabilities $\alpha(t)$ and $\beta((t-x) / h)$

Our objective is to minimize the average cost per time unit $g(M, T)$ over an infinite time horizon by optimizing the decision variables $M$ and $T$ of the maintenance policy, i.e.,

$$
\min _{M, T}\{g(M, T): T>0, M \in \mathbb{N}\}
$$

\section{Notation}

$X \quad$ : Continuous non-negative random variable representing the system's time to defect

$H \quad$ : Continuous non-negative random variable representing the system's delay time

$F_{X}(\cdot) \quad$ : Cumulative distribution function for the random variable $X$

$F_{H}(\cdot) \quad$ : Cumulative distribution function for the random variable $H$

$f_{X}(\cdot) \quad$ : Probability density function for the random variable $X$

$f_{H}(\cdot) \quad$ : Probability density function for the random variable $H$ 

$M-1$ : Maximum number of inspections before preventive system maintenance
$T \quad:$ Fixed inspection interval length
$c_{i} \quad:$ Cost per inspection
$c_{p} \quad:$ Cost for preventive maintenance
$c_{c} \quad:$ Cost for corrective maintenance
$\alpha(\cdot) \quad$ : Non-constant probability of a false positive inspection
$\beta(\cdot) \quad$ : Non-constant probability of a false negative inspection

\section{Model analysis}

This section presents the analysis of the optimization model from Equation (1). Again, we focus on the true model here, since the analysis of the approximate model can directly be derived from the analysis below, i.e., the probabilities of inspection errors are constant. Furthermore, we would like to remark that the analysis holds for any functional form of $\alpha(t)$ and $\beta((t-x) / h)$. We derive an exact expression for the average cost per time unit $g(M, T)$ in Section 3.1, and present our optimization procedure in Section 3.2.

\subsection{Evaluation procedure}

Corrective and preventive maintenance actions constitute renewal points. We define a renewal cycle as the time between two such successive renewal points. From renewal theory (Ross, 1983) we know that the average cost over one renewal cycle equals the average cost over an infinite time horizon $g(M, T)$. We use these properties to obtain the expression for $g(M, T)$. Then,

$$
g(M, T)=\frac{C(M, T)}{L(M, T)},
$$

where $C(M, T)$ represents the expected renewal cycle cost and $L(M, T)$ represents the expected renewal cycle length both under the decision variables $M$ and $T$. We will refer to the tuple of decision variables $(M, T)$ as a maintenance policy. The derivation for the terms $C(M, T)$, and $L(M, T)$ is based on various event paths that may occur in a cycle. We study these event paths, and derive the corresponding probability expressions, which yields the expression for $g(M, T)$.

Event Path Type 1 (E1). The system survives without any defect occurrence until time $M T$, at which the cycle ends. This implies that its time to defect has to exceed $M T$, and that no false 
positives occur at any of the inspections before $M$. We refer to this event path as EventPath(1). Because the inspections are done every $T$ time units, false positives do not occur at inspections $1, \ldots, M-1$, corresponding to the times $T, \ldots,(M-1) T$. Hence, the probability of false positives is evaluated at these time epochs. Since the probability of false positives is non-constant, a product series is included from inspections 1 to $M-1$. The probability expression for the Event Path Type 1 equals

$$
\pi_{1}=\int_{M T}^{\infty} \prod_{n=1}^{M-1}(1-\alpha(n T)) f_{X}(x) d x=\left(1-F_{X}(M T)\right) \prod_{n=1}^{M-1}(1-\alpha(n T)) .
$$

Event Path Type 2 (E2). A false positive occurs at inspection $j \in\{1, \ldots, M-1\}$, thereby ending the cycle. This implies that the time to defect exceeds time $j T$, and before inspection $j$ no false positives have occurred. This event path is referred to as EventPath $(2, j)$. The corresponding probability is:

$\pi_{2, j}=\int_{j T}^{\infty} \prod_{n=1}^{j-1}(1-\alpha(n T)) \alpha(j T) f_{X}(x) d x=\left(1-F_{X}(j T)\right) \alpha(j T) \prod_{n=1}^{j-1}(1-\alpha(n T)), \quad j \in\{1, \ldots, M-1\}$.

Event Path Type 3 (E3). The system becomes defective at time $x$, in an interval $[(i-1) T, i T)$, characterized by inspection $i \in\{1, \ldots, M\}$, and fails in this very interval, i.e., a false negative cannot occur. This implies that its delay time lies in the interval $[0, i T-x)$. Before defect arrival, no false positives occur. We denote this event path by EventPath $(3, i)$. The probability of EventPath $(3, i)$ is given by

$$
\pi_{3, i}=\int_{(i-1) T}^{i T} \prod_{n=1}^{i-1}(1-\alpha(n T)) \int_{0}^{i T-x} f_{H}(h) d h f_{X}(x) d x, \quad i \in\{1, \ldots, M\} .
$$

Event Path Type 4 (E4). The system becomes defective in an interval $[(i-1) T, i T)$ characterized by inspection $i \in\{1, \ldots, M-1\}$. In contrast to the third type of event paths, the system does not fail in this interval, but fails in an interval $[j T,(j+1) T), j \in\{i, \ldots, M-1\}$. We refer to this event path as EventPath $(4, i, j)$. False negatives may occur at inspections $i$ up to $j$, and we include a product series due to the non-constant false negative probability. Since the false negatives can only occur at inspection instances, we consider $k T$ in the product series, where $k=i, \ldots, j$. No false positives occur before inspection $i$. Note that the event paths of type 4 are characterized by the inspections $i$ and $j$, and therefore we obtain the probability expression:

$$
\begin{array}{r}
\pi_{4, i, j}=\int_{(i-1) T}^{i T} \prod_{n=1}^{i-1}(1-\alpha(n T)) \int_{j T-x}^{(j+1) T-x} \prod_{k=i}^{j} \beta\left(\frac{k T-x}{h}\right) f_{H}(h) d h f_{X}(x) d x, \\
i \in\{1, \ldots, M-1\}, j \in\{i, \ldots, M-1\} .
\end{array}
$$


Event Path Type 5 (E5). The system becomes defective in an interval $[(i-1) T, i T)$, where $i \in\{1, \ldots, M-1\}$, and no false positives occur before defect arrival. The system's defect is revealed at inspection $j \in\{i, \ldots, M-1\}$, denoting that the system's delay time has to exceed $j T-x$. This event path is referred to as EventPath $(5, i, j)$. Note that the detection of the defect occurs at time $j T$. This means that for inspections $i$ up to $j-1$, false negatives occur with a non-constant probability. The corresponding probability of EventPath $(5, i, j)$ corresponds to

$$
\begin{array}{r}
\pi_{5, i, j}=\int_{(i-1) T}^{i T} \prod_{n=1}^{i-1}(1-\alpha(n T)) \int_{j T-x}^{\infty} \prod_{k=i}^{j-1} \beta\left(\frac{k T-x}{h}\right) \\
\left(1-\beta\left(\frac{j T-x}{h}\right)\right) f_{H}(h) d h f_{X}(x) d x \\
i \in\{1, \ldots, M-1\}, j \in\{i, \ldots, M-1\}
\end{array}
$$

Event Path Type 6 (E6). The system becomes defective at time $x$ in $[(i-1) T, i T)$, where $i \in\{1, \ldots, M\}$, and remains defective until the system is renewed at time $M T$. Before the defect arrives, no false positives occur. From the defect arrival at time $x$ to $M T$, the system remains defective. This implies that inspections $i$ up to $M-1$ undergo false negatives. We denote this event path by EventPath $(6, i)$, with corresponding probability

$$
\pi_{6, i}=\int_{(i-1) T}^{i T} \prod_{n=1}^{i-1}(1-\alpha(n T)) \int_{M T-x}^{\infty} \prod_{k=i}^{M-1} \beta\left(\frac{k T-x}{h}\right) f_{H}(h) d h f_{X}(x) d x, \quad i \in\{1, \ldots, M\} .
$$

From the six different types of event paths, we derive the expression for the expected renewal cycle costs $C(M, T)$. For E3 and E4 corrective maintenance costs $c_{c}$ are incurred, since these event path types end in system failure. Furthermore, for E3 and E4, $i-1$ and $j$ inspections are done, respectively. Consequently the cost of $\mathrm{E} 3$ corresponds to $(i-1) c_{i}+c_{c}$, and for $\mathrm{E} 4$ the cost is given by $j c_{i}+c_{c}$. Since all other types of event paths, E1, E2, E5, and E6 end in preventive maintenance, cost $c_{p}$ are incurred. For these event types, the number of inspections and the total inspection cost can be determined. By the expressions for the probabilities of the event paths, the expected cycle cost $C(M, T)$ are obtained by summing the event paths over all possible $i$ and $j$ :

$$
\begin{aligned}
C(M, T) & =\left((M-1) c_{i}+c_{p}\right) \pi_{1}+\sum_{j=1}^{M-1}\left(j c_{i}+c_{p}\right) \pi_{2, j}+\sum_{i=1}^{M}\left((i-1) c_{i}+c_{c}\right) \pi_{3, i}+\sum_{i=1}^{M-1} \sum_{j=i}^{M-1}\left(j c_{i}+c_{c}\right) \pi_{4, i, j} \\
& +\sum_{i=1}^{M-1} \sum_{j=i}^{M-1}\left(j c_{i}+c_{p}\right) \pi_{5, i, j}+\sum_{i=1}^{M}\left((M-1) c_{i}+c_{p}\right) \pi_{6, i} .
\end{aligned}
$$

We derive the expression for the expected renewal cycle length $L(M, T)$ in a similar way to $C(M, T)$. For E3 and E4, the cycle ends in system failure. Therefore, the cycle length depends on 
the realizations of $x$ and $h$, and involves the integration over $x$ and $h$. The other types of event paths renew at the time epoch of preventive maintenance (at an inspection or at $M T$ ). When summing over all possible values of $i$ and $j$ for all event paths, we obtain

$$
\begin{aligned}
& L(M, T)=M T \pi_{1}+\sum_{j=1}^{M-1} j T \pi_{2, j}+\sum_{i=1}^{M} \int_{(i-1) T}^{i T} \prod_{n=1}^{i-1}(1-\alpha(n T)) \int_{0}^{i T-x}(x+h) f_{H}(h) d h f_{X}(x) d x \\
& \quad+\sum_{i=1}^{M-1} \sum_{j=i}^{M-1} \int_{(i-1) T}^{i T} \prod_{n=1}^{i-1}(1-\alpha(n T)) \int_{j T-x}^{(j+1) T-x} \prod_{k=i}^{j} \beta\left(\frac{k T-x}{h}\right)(x+h) f_{H}(h) d h f_{X}(x) d x \\
& \quad+\sum_{i=1}^{M-1} \sum_{j=i}^{M-1} j T \pi_{5, i, j}+\sum_{i=1}^{M} M T \pi_{6, i} .
\end{aligned}
$$

\subsection{Optimization procedure}

For optimizing our problem with non-constant probabilities, cf. Equation (1), we enumerate maintenance policies characterized by $(M, T)$. We restrict ourselves to $M \in\left\{1, \ldots, M_{\max }\right\}$, with $M_{\max }$ corresponding to the enumeration upper bound. Then, we perform the following procedure for each $M \in\left\{1, \ldots, M_{\max }\right\}$.

We enumerate the inspection interval lengths $T_{M} \in\left\{\delta, 2 \delta, \ldots, k_{\max }(E[X]+E[H])\right\}$. $\delta$ corresponds to the stepsize and $k_{\max }(E[X]+E[H])$ denotes the upper bound of the considered inspection interval length $T_{M}$, with $E[\cdot]$ representing the expected value of a random variable. We denote the optimal inspection interval length over all $T_{M} \in\left\{\delta, 2 \delta, \ldots, k_{\max }(E[X]+E[H])\right\}$ by $\tilde{T}_{M}$. Next, we improve our result by doing a local search around $\tilde{T}_{M}$. That is, we define a new search space, bounded from below by $\underline{T}_{M}=\tilde{T}_{M}-\delta$ and bounded from above by $\bar{T}_{M}=\tilde{T}_{M}+\delta$. Furthermore, we refine our stepsize to $\Delta=\left(\bar{T}_{M}-\underline{T}_{M}\right) / \lambda$, with $\lambda+1$ defining the number of the steps. We

enumerate all $T_{M} \in\left(\underline{T}_{M}, \bar{T}_{M}\right)$ with stepsize $\Delta$, and we denote the optimum $T_{M} \in\left(\underline{T}_{M}, \bar{T}_{M}\right)$ by $T_{M}^{\prime}$

We perform the above procedure for each $M \in\left\{1, \ldots, M_{\max }\right\}$, and subsequently select the optimum maintenance policy over all $M \in\left\{1, \ldots, M_{\max }\right\}$. We denote this optimal policy by $\left(M^{*}, T^{*}\right)$. In case we consider the approximate model with constant probabilities, we apply the same procedure and we obtain the optimal maintenance policy $(\widehat{M}, \widehat{T})$.

Example 1. Let $c_{i}=\$ 100, c_{p}=\$ 1,000, c_{c}=\$ 100,000, \alpha_{0}=0.05, u_{\alpha}=0.50, a=900$ days, $\beta_{0}=$ 0.05, $\eta=2.0, \gamma=5.0, M_{\max }=40, \delta=(E[X]+E[H]) / 50, k_{\max }=2$, and $\lambda=50$. Furthermore, we let $X$ and $H$ (in days) be Weibull distributed such that $E[X]=900$ days, $E[H]=100$ days, 
$C V_{X}=0.50$ and $C V_{H}=0.50$, where $C V_{X}$ and $C V_{H}$ denote the coefficient of variation for the distribution of the time to defect and delay time, respectively.

By applying the above procedure, we obtain: $\left(M^{*}, T^{*}\right)=(6,52.00)$ with corresponding costs $g\left(M^{*}, T^{*}\right)=\$ 7.99$ per day. In case we would have perfect inspections, we would obtain $\left(M^{*}, T^{*}\right)=$ $(15,37.60)$ with costs $g\left(M^{*}, T^{*}\right)=\$ 5.87$ per day.

Remark 1. Suppose we have a multi-component system that is inspected every $\zeta$ time units and a component can only be inspected on integer multiples of $\zeta$, i.e., $d \zeta, d \in \mathbb{N}_{+}$. Note that in this case, the inspection schedule does not restart upon system failure. Then, our model can be used as an approximation for evaluating the costs associated to a policy that inspects the component each $T_{M}=d \zeta$ time units, and preventively replaces the component after $M$ inspections. In this case, the optimization procedure would consider $T_{M} \in\left\{\zeta, 2 \zeta, \ldots,\left\lfloor k_{\max }(E[X]+E[H]) / \zeta\right\rfloor\right\}$. If a failure occurs we perform the first inspection between $(d-1) T_{M}$ and $d T_{M}$ time units after the failure. Subsequently we follow the inspection schedule with interval length $T_{M}$, see Figure 2 (where the cross denotes the time of component failure). The effects of the approximation will be limited, since the lifetime of a component is usually relatively long compared to its inspection interval and thus the rounding effect becomes small.

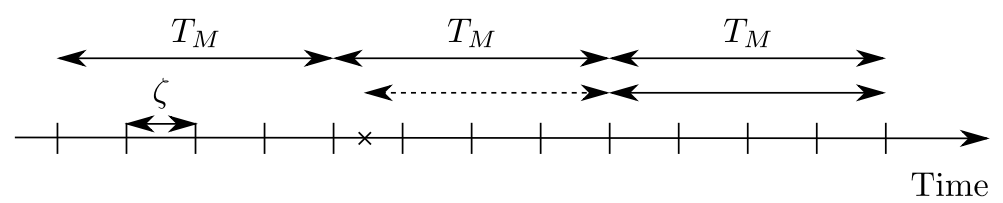

Figure 2: Our model as approximation to a multi-component system approach $(\mathrm{d}=4)$

\section{Comparing the true and approximate model}

The difference between the true and approximate model is the way of modeling the probability of false positives and false negatives. The former model entails a detailed modeling of $\alpha(t)$ and $\beta((t-x) / h)$, while the latter has a simpler modeling because it considers constant probabilities instead of non-constant functions. The benefit of using the approximate model is that it is easier to understand and easier to implement. It requires the user to estimate the constant probability of false positives and false negatives and subsequently the model determines the optimal maintenance policy $(\widehat{M}, \widehat{T})$. 
In this section, we are interested in comparing the true model to the approximate model. To achieve this, we should set the functions for the true model appropriately, as well as the constant probabilities for the approximate model. We consider that the true model is the reality, and that the approximate model is an approximation that uses simpler modeling. This implies that the functions $\alpha(t)$ and $\beta((t-x) / h)$ describe the true behavior, and we need to set the constant probabilities in the approximate model in a fair way, i.e., such that both models can be compared fairly.

\subsection{Comparison procedure}

The true and approximate models are compared under their optimal policies such that the fraction of false positives and false negatives that occur over an infinite time horizon are equal in both models. We remark that the comparison method works for any functional forms of $\alpha(t)$ and $\beta((t-x) / h)$.

Our first step is to optimize the true model for given functions for $\alpha(t)$ and $\beta((t-x) / h)$. The fraction of false positives and false negatives that occur over an infinite time horizon, under the true model, depend on the maintenance policy $(M, T)$ chosen. We denote the fraction at which a false positive occurs over an infinite time horizon by $\mu_{\alpha}(M, T)$. This $\mu_{\alpha}(M, T)$ is determined over all inspections that are executed - under policy $(M, T)$ - when the component is in the normal state. Similarly, $\mu_{\beta}(M, T)$ corresponds to the fraction at which false negatives are generated, under policy $(M, T)$ over an infinite time horizon, and based on all inspections that are done when the system is in its defective state.

The optimization of the true model results in an optimal policy $\left(M^{*}, T^{*}\right)$ and in fractions $\mu_{\alpha}\left(M^{*}, T^{*}\right)$ and $\mu_{\beta}\left(M^{*}, T^{*}\right)$ for false positives and false negatives, respectively. We want to compare the true and approximate model such that the fraction at which false positives and false negatives occur are the same for both models. Under the approximate model, the probabilities of inspection errors are constant and are unaffected by the maintenance policy. Therefore, setting $\widehat{\alpha}(t)=\mu_{\alpha}\left(M^{*}, T^{*}\right)$ and $\widehat{\beta}((t-x) / h)=\mu_{\beta}\left(M^{*}, T^{*}\right)$ for the approximate model ensures that the fractions at which false positives and false negatives occur are the same for both models. Recall that hats above variable denote that we consider constant probabilities of false positives and false negatives. Similarly, $\widehat{g}(M, T)$ corresponds to the costs of a maintenance policy $(M, T)$ under constant probabilities, and is considered to be a costs approximation. Our procedure for comparing the true and approximate model is illustrated in Figure 3.

We remark that no inspections are done when $M=1$, since this corresponds to an age-based maintenance policy. Consequently, $\mu_{\alpha}(M, T)$ and $\mu_{\beta}(M, T)$ are not defined for $M=1$. Hence, we 


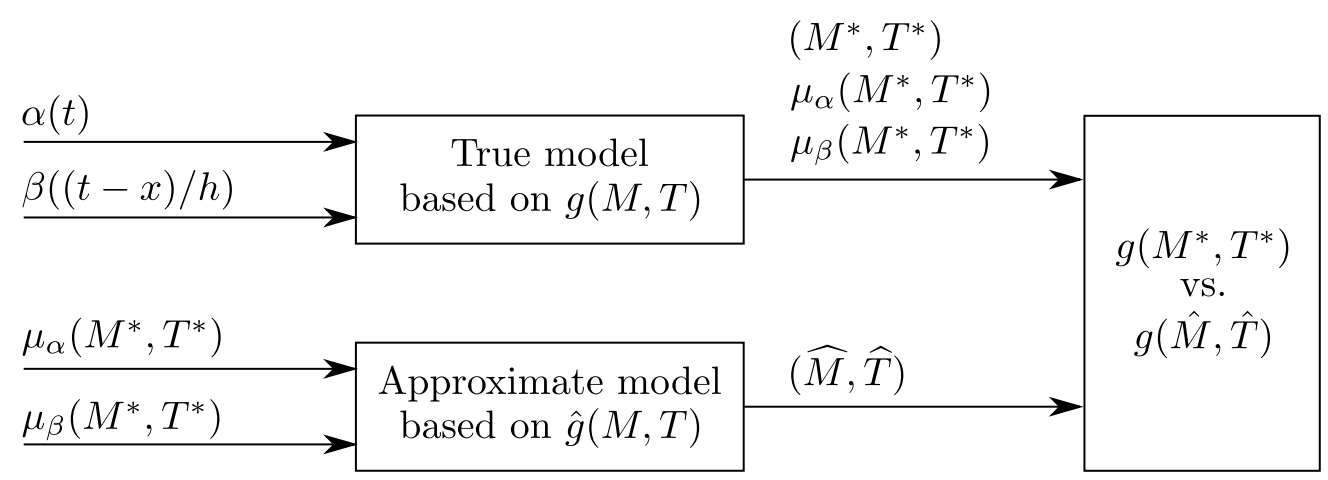

Figure 3: Comparing the true and approximate model

assume that if $M^{*}=1$, then $\widehat{M}=1$ as well, and consequently $T^{*}=\widehat{T}$.

\subsection{Fraction of false positives and false negatives}

In this section, we derive expressions for $\mu_{\alpha}(M, T)$ and $\mu_{\beta}(M, T)$. The fraction $\mu_{\alpha}(M, T)$ is equal to the average number of false positives $z_{\alpha}(M, T)$ per time unit, divided by the total number of executed inspections per time unit $t_{\alpha}(M, T)$ when the system is in its normal state, i.e., $\mu_{\alpha}(M, T)=$ $z_{\alpha}(M, T) / t_{\alpha}(M, T)$. From renewal theory (Ross, 1983), it follows that $z_{\alpha}(M, T)$ and $t_{\alpha}(M, T)$ equal the average number of false positives and the expected total number of inspections done when the system is in its normal state, over one renewal cycle, respectively. That is, $z_{\alpha}(M, T)=$ $Z_{\alpha}(M, T) / L(M, T)$, and $t_{\alpha}(M, T)=T_{\alpha}(M, T) / L(M, T)$, where $Z_{\alpha}(M, T)$ denotes the expected number of false positives in a renewal cycle, $T_{\alpha}(M, T)$ the expected total number of inspections in a renewal cycle when the system is in its normal state, and $L(M, T)$ represents the expected renewal cycle length. Rewriting the expression for $\mu_{\alpha}(M, T)$ yields

$$
\mu_{\alpha}(M, T)=\frac{Z_{\alpha}(M, T)}{T_{\alpha}(M, T)}, \forall M \geq 2
$$

To derive expressions for $Z_{\alpha}(M, T)$ and $T_{\alpha}(M, T)$ we use the event path types from Section 3.1. The average number of false positives that occur in a renewal cycle $Z_{\alpha}(M, T)$ equals the sum of the probabilities of each possible EventPath $(2, j)$. Hence, $Z_{\alpha}(M, T)=\sum_{j=1}^{M-1} \pi_{2, j}$. The total expected number of inspections $T_{\alpha}(M, T)$ is derived by considering all event paths from Section 3.1. Multiplying the probability of an event path by the corresponding number of inspections (when the system is in its normal state), and summing over all possible $i$ and $j$, we obtain

$$
T_{\alpha}(M, T)=(M-1) \pi_{1}+\sum_{j=1}^{M-1} j \pi_{2, j}+\sum_{i=1}^{M}(i-1)\left(\pi_{3, i}+\pi_{6, i}\right)+\sum_{i=1}^{M-1} \sum_{j=i}^{M-1}(i-1)\left(\pi_{4, i, j}+\pi_{5, i, j}\right) .
$$


Similar to $\mu_{\alpha}(M, T)$, we have that $\mu_{\beta}(M, T)$ equals the average number of false negatives per time unit $z_{\beta}(M, T)$, divided by the total number of inspections done per time unit $t_{\beta}(M, T)$ when the system is defective. Consequently, we derive

$$
\mu_{\beta}(M, T)=\frac{Z_{\beta}(M, T)}{T_{\beta}(M, T)}, \forall M \geq 2
$$

with $Z_{\beta}(M, T)$ the average number of false negatives per renewal cycle, and $T_{\beta}(M, T)$ the expected total number of inspections when the system is defective over one renewal cycle.

To derive the expected number of false negatives in a renewal cycle, $Z_{\beta}(M, T)$, we use the event path types E4-E6 from Section 3.1. Each of these event paths occurs with probability $\pi_{4, i, j}, \pi_{5, i, j}$ and $\pi_{6, i}$ respectively. The expected number of false negatives in a renewal cycle is given by

$$
Z_{\beta}(M, T)=\sum_{i=1}^{M-1} \sum_{j=i}^{M-1}(j-i+1) \pi_{4, i, j}+\sum_{i=1}^{M-1} \sum_{j=i}^{M-1}(j-i) \pi_{5, i, j}+\sum_{i=1}^{M-1}(M-i) \pi_{6, i} .
$$

Similarly, we derive the expression for the expected total number of inspections when the system is defective:

$$
T_{\beta}(M, T)=\sum_{i=1}^{M-1} \sum_{j=i}^{M-1}(j-i+1)\left(\pi_{4, i, j}+\pi_{5, i, j}\right)+\sum_{i=1}^{M-1}(M-i) \pi_{6, i}=Z_{\beta}(M, T)+\sum_{i=1}^{M-1} \sum_{j=i}^{M-1} \pi_{5, i, j} .
$$

Example 1 (Continued). We obtain $\mu_{\alpha}\left(M^{*}, T^{*}\right)=0.13$ and $\mu_{\beta}\left(M^{*}, T^{*}\right)=0.26$, and consequently $(\widehat{M}, \widehat{T})=(3,71.20)$ with $g(\widehat{M}, \widehat{T})=\$ 9.51$ per day corresponding to the true costs. Note that these costs are substantially higher compared to $g\left(M^{*}, T^{*}\right)=\$ 7.99$ per day.

\section{Computational study}

In this section we present our computational study, in which we focus on six main factors. We define a base instance (corresponding to Example 1), and then vary each factor to a high and low level. This implies that we create a testbed consisting of $2 \times 6+1=13$ instances. We compute the optimal policies for the true and approximate model, $\left(M^{*}, T^{*}\right)$ and $(\widehat{M}, \widehat{T})$, respectively. Our main interest is the relative cost difference $\Delta g$ between the optimal policy of the true model and the optimal policy of the approximate model. Note that we compare both policies under the true cost function $g(M, T)$. Thus, the cost difference $\Delta g$ is defined by

$$
\Delta g=\frac{g(\widehat{M}, \widehat{T})-g\left(M^{*}, T^{*}\right)}{g\left(M^{*}, T^{*}\right)} .
$$




\section{$5.1 \quad$ Testbed}

We relate the false positive probability to the time $t$ that the system has been operating in its normal state, counted from the last renewal point, see Section 2. We assume that the probability of a false positive is related to $t$ in a piecewise manner, for simplicity and because ours is a first attempt to model such a non-constant probability $\alpha(t)$. Consequently, we assume that $\alpha(t)$ increases to a threshold $a$, and from this point stays constant, see Figure 4. Then,

$$
\alpha(t)=\left\{\begin{array}{ll}
\alpha_{0}+\left(u_{\alpha} t\right) / a & \text { if } t \leq a \\
\alpha_{0}+u_{\alpha} & \text { otherwise }
\end{array},\right.
$$

where $\alpha_{0}$ represents the false positive probability that does not depend on the time $t$. For the probability of false negatives, we follow the literature on the Probability of Detection (POD). Since $\beta=1-P O D$, and the relationship between the POD and system degradation is best modeled by a log odds distribution (Berens, 1989; Georgiou, 2006), we relate the failure progress to the probability of false negatives in log odds terms as well. Hence,

$$
\beta\left(\frac{t-x}{h}\right)=\beta_{0}+\frac{1-\beta_{0}}{1+e^{\gamma+\eta \ln \left(\frac{t-x}{h}\right)}}, \quad \text { if } x \leq t \leq x+h
$$

where $\eta \geq 0$ and $\gamma$ are shape parameters, and $\beta_{0}$ corresponds to the false negative probability that does not depend on the failure progress. For further details on the log-odds distribution, see Georgiou (2006). Figure 4 illustrates the proposed functions for the probability of false positives related to $t$, and for the probability of false negatives related to the failure progress $(t-x) / h$, under various parameter settings.
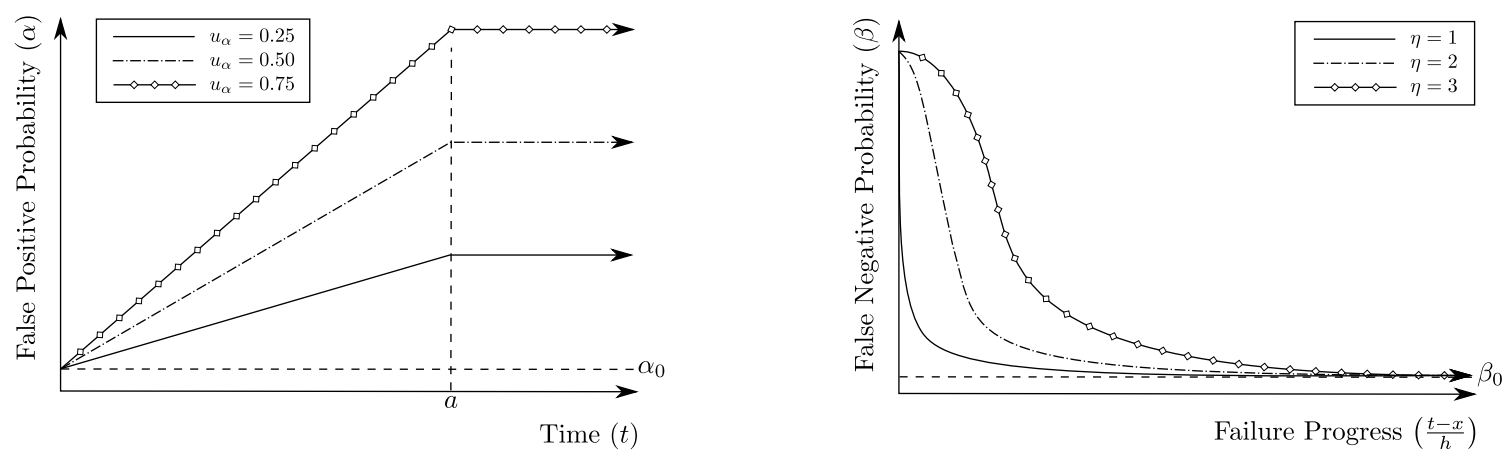

Figure 4: Functions $\alpha(t)$ and $\beta((t-x) / h)$ for various parameter settings

We explore the effects of six factors: the corrective maintenance $\operatorname{costs} c_{c}$, the inspection cost parameter $c_{i}$, the bandwidth of the non-constant probability of false positives (and defining the 
slope) $u_{\alpha}$, the shape parameter $\eta$ for the probability of false negatives, the coefficient of variation of the time to defect $C V_{X}$, and the coefficient of variation of the delay time $C V_{H}$. Note that a decrease in $\gamma$ yields a similar result as an increase in $\eta$, as the $\ln (\cdot)$ term is negative. We use Weibull distributions in our computational study for the time to defect and the delay time. We find the shape and scale parameters of the Weibull distributions by first searching for the shape parameter that yields the corresponding coefficient of variation. We start by restricting our focus to the coefficient of variation, since this is solely determined by the shape parameter. Subsequently, we determine the scale parameter to obtain the given expected time to defect $E[X]$ and delay time $E[H]$. All factor choices are presented in Table 4, and the values of our base instance are listed in Table 5 .

\begin{tabular}{llllll}
\hline$c_{c}(\$)$ & $c_{i}(\$)$ & $u_{\alpha}$ & $\eta$ & $C V_{X}$ & $C V_{H}$ \\
\hline 50,$000 ; 100,000 ; 200,000$ & $50 ; 100 ; 200$ & $0.25 ; 0.50 ; 0.75$ & $1.0 ; 2.0 ; 3.0$ & $0.25 ; 0.50 ; 1.0$ & $0.25 ; 0.50 ; 1.0$ \\
\hline
\end{tabular}

Table 4: Factor choices

\begin{tabular}{|c|c|c|c|c|c|c|c|c|c|c|c|c|}
\hline$E[X]$ (days) & $E[H]$ (days) & $C V_{X}$ & $C V_{H}$ & $c_{i}(\$)$ & $c_{p}(\$)$ & $c_{c}(\$)$ & $\alpha_{0}$ & $u_{\alpha}$ & $a$ (days) & $\beta_{0}$ & $\eta$ & $\gamma$ \\
\hline 900 & 100 & 0.50 & 0.50 & 100 & 1,000 & 100,000 & 0.05 & 0.50 & 900 & 0.05 & 2.0 & 5 \\
\hline
\end{tabular}

Table 5: Values of factors and parameters of the base instance

Finally, we use the following parameter settings for the optimization procedure: $M_{\max }=40$, $k_{\max }=2$, and $\lambda=50$. These settings provide sufficient detail in the considered inspection interval lengths without unnecessarily increasing the computation times.

\subsection{Results and managerial insights}

Our numerical results are presented in Table 6 . When $M^{*}=\widehat{M}=1$, the expressions $\mu_{\alpha}$ and $\mu_{\beta}$ are not defined, and are denoted by a dash in Table 6 . We will first study the results of the true model (columns 2-5), and secondly we will discuss the results of the approximate model (columns 8-11). Finally, we elaborate on the cost differences that we observe in the last column.

Our first observations are made with respect to the optimal maintenance policies $\left(M^{*}, T^{*}\right)$. We explore the factors considering the inspection characteristics, i.e., $c_{i}, u_{\alpha}$ and $\eta$. Subsequently, 


\begin{tabular}{|c|c|c|c|c|c|c|c|c|c|c|c|c|}
\hline & & $\left(M^{*}, T^{*}\right)$ & $L\left(M^{*}, T^{*}\right)$ & $M^{*} T^{*}$ & $g\left(M^{*}, T^{*}\right)$ & $\mu_{\alpha}$ & $\mu_{\beta}$ & $(\widehat{M}, \widehat{T})$ & $L(\widehat{M}, \widehat{T})$ & $\widehat{M} \widehat{T}$ & $g(\widehat{M}, \widehat{T})$ & $\Delta g(\%)$ \\
\hline \multirow[t]{3}{*}{$c_{i}$} & 50 & $(10$, & 239.57 & 416.01 & 6.97 & $\mid 0.14$ & 0.27 & $(6,47.20)$ & 174.27 & 283.21 & 9.47 & 35.72 \\
\hline & 100 & $(6,52.00)$ & 232.05 & 312.01 & 7.99 & 0.13 & 0.26 & $(3,71.20)$ & 176.74 & 213.61 & 9.51 & 19.00 \\
\hline & 200 & $(1,154.40)$ & 154.28 & 154.40 & 9.01 & & & $(1,154.40)$ & 154.28 & 154.40 & 9.01 & 0.00 \\
\hline \multirow[t]{3}{*}{$u_{\alpha}$} & 0.25 & & 6.32 & & 7.69 & 0.10 & 0.27 & $(4,5 !$ & 195.82 & 236.81 & 9.32 & 21.3 \\
\hline & 0.5 & $(6,52.00)$ & 232.05 & 312.01 & 7.99 & 0.13 & 0.26 & $(3,71.20)$ & 176.74 & 213.61 & 9.51 & 19.00 \\
\hline & 0.75 & $(5,56.80)$ & 212.23 & 284.01 & 8.25 & 0.16 & 0.26 & $(2,93.60)$ & 164.74 & 187.20 & 9.46 & 14.62 \\
\hline \multirow[t]{3}{*}{$\eta$} & 1 & & & 326.41 & 7.64 & 0.13 & 0.11 & & & 331.21 & 8.73 & 14.21 \\
\hline & 2 & $(6,52.00)$ & 232.05 & 312.01 & 7.99 & 0.13 & 0.26 & $(3,71.20)$ & 176.74 & 213.61 & 9.51 & 19.00 \\
\hline & 3 & $(5,53.60)$ & 214.32 & 268.01 & 8.43 & 0.12 & 0.41 & $(1,154.40)$ & 154.28 & 154.40 & 9.01 & 6.91 \\
\hline \multirow[t]{3}{*}{$c_{c}$} & 50,000 & $(5,68.00)$ & 261.80 & 10.01 & 6.76 & 0.14 & 0.24 & $(3,89.60)$ & 216.62 & 268.81 & 7.84 & 15.90 \\
\hline & 100,000 & $(6,52.00)$ & 232.05 & 312.01 & 7.99 & 0.13 & 0.26 & $(3,71.20)$ & 176.74 & 213.61 & 9.51 & 19.00 \\
\hline & 200,000 & $(7,40.80)$ & 206.11 & 285.61 & 9.41 & 0.12 & 0.29 & $(3,57.60)$ & 145.91 & 172.80 & 11.49 & 22.0 \\
\hline \multirow[t]{3}{*}{$C V_{X}$} & 0.25 & $(1,340.01)$ & & 340.01 & 3.57 & & & $(1,340.01)$ & 339.90 & 340.01 & 3.57 & 0.00 \\
\hline & 0.5 & $(6,52.00)$ & 232.05 & 312.01 & 7.99 & 0.13 & 0.26 & $(3,71.20)$ & 176.74 & 213.61 & 9.51 & 19.00 \\
\hline & 1 & $(27,28.80)$ & 184.90 & 777.61 & 12.54 & 0.13 & 0.31 & $(40,23.20)$ & 115.76 & 928.02 & 21.17 & $68.8 ?$ \\
\hline \multirow[t]{3}{*}{$C V_{H}$} & 0.25 & $(7,57.60)$ & 270.14 & 403.21 & 6.46 & 0.15 & 0.23 & $(3,78.40)$ & 189.84 & 235.20 & 8.49 & 31.51 \\
\hline & 0.5 & $(6,52.00)$ & 232.05 & 312.01 & 7.99 & 0.13 & 0.26 & $(3,71.20)$ & 176.74 & 213.61 & 9.51 & 19.00 \\
\hline & 1 & $(3,63.20)$ & 171.14 & 189.60 & 10.70 & 0.10 & 0.30 & $(1,144.80)$ & 144.59 & 144.80 & 10.77 & 0.67 \\
\hline
\end{tabular}

Table 6: Experiment results 
we will address the other three factors. We see that $M^{*}$ decreases, $T^{*}$ increases, and $M^{*} T^{*}$ and $L\left(M^{*}, T^{*}\right)$ decrease, when inspections are more expensive or are more prone to false positives $\left(u_{\alpha}\right.$ increases). This phenomenon indicates that inspections are less attractive when the inspection cost or the false positive probability (in terms of $u_{\alpha}$ ) increases. If the false negative probability increases (in terms of $\eta$ ), we observe a decrease in $M^{*}, M^{*} T^{*}$, and in $L\left(M^{*}, T^{*}\right.$ ), while $T^{*}$ remains roughly equal. This illustrates that preventive maintenance is done earlierin order to prevent the system from failing. Next, we continue by studying the changes in $c_{c}, C V_{X}$, and $C V_{H}$. We observe that more inspections are scheduled and that the time of preventive maintenance decreases, as the cost of corrective maintenance $c_{c}$ increases, i.e., $M^{*}$ increases, $T^{*}$ decreases, and $M^{*} T^{*}$ and $L\left(M^{*}, T^{*}\right)$ decrease. Failure of the system is more costly, and consequently more inspections and earlier preventive maintenance are scheduled in an optimal maintenance policy, in order to prevent the costly system failure. Inspections are scheduled more frequently, in case the time to defect's distribution is more variable, i.e., $C V_{X}$ increases. Furthermore, we observe that preventive maintenance becomes unattractive in the extreme case of an exponential distribution $\left(C V_{X}=1.0\right)$, because preventive maintenance adds little value when the system is in its normal state due to the memoryless property of the exponential distribution. That is, the system is in the normal state most of the time (in expectation), and in this state preventive maintenance adds very little value. Finally, we address an increase in the variation of the delay time, i.e., an increase in $C V_{H}$. We see that an optimal maintenance policy focuses more on preventive maintenance, as $M^{*}, L\left(M^{*}, T^{*}\right)$ and $M^{*} T^{*}$ decrease. This behavior occurs because, a higher variability in the delay time increases the probability of failure once the system has reached the defective state, and consequently we focus more on preventive maintenance in order to prevent this failure from occurring.

Let us now consider the effect of the factor changes on the costs of true model. We observe that the costs $g\left(M^{*}, T^{*}\right)$ increase when the cost of inspections increases, and when the quality of inspections decreases. Furthermore, we see that the costs increase in case the cost of corrective maintenance increases. Finally, we observe that a higher variability in the time to defect and the delay time's distribution also induces a cost increase. We do not further elaborate on these results, as they are in line with our expectation and intuition.

For the optimal policies $(\widehat{M}, \widehat{T})$, we observe that the approximate model prefers preventive maintenance over inspections, when the inspections are more expensive, when the inspection quality (in terms of $u_{\alpha}$ or $\eta$ ) decreases, when the corrective maintenance costs increase, and when the delay time is more variable. Furthermore, we see that a higher variability in the distribution of the time 
to defect $\left(C V_{X}\right.$ increases) results in maintenance policies under which inspections are scheduled more frequently and preventive maintenance is scheduled earlier. These phenomena align with the ones seen at the true model, and therefore we will not elaborate on this any further. Moreover, we observe the same cost effects as the true model when considering $g(\widehat{M}, \widehat{T})$. However, some exceptions occur when few inspections are scheduled under the approximate model (low $\widehat{M}$ ). In these cases, the approximate model resembles the true model, because few or no inspections are done, and thus the cost difference $\Delta g$ is typically small(er).

In this final part of our discussion, we focus on the cost differences $\Delta g$. For $c_{i}, u_{\alpha}, c_{c}$ and $C V_{X}$, we observe that the cost difference $\Delta g$ increases when the number of inspections per time unit increases, i.e., when the inspection frequencies $1 / T^{*}$ and $1 / \widehat{T}$ increase. In this case, the constant probability approximation is applied to more inspections per time unit, thereby increasing $\Delta g$. For

false negatives, when $\eta$ increases from 1 to 2 , Table 6 shows that $T^{*}$ and $\widehat{M}$ decrease. As a result, relatively many inspections are planned before preventive maintenance for the true model compared to the approximate model, and thus $\Delta g$ increases. However, we observe that $\Delta g$ decreases when $\eta$ increases further from 2 to 3 , because the result of the approximate model $(\widehat{M}, \widehat{T})$ coincides with the optimal policy of the true model under $M^{*}$. Consequently, this reduces $\Delta g$. An increase in the coefficient of variation of the delay time $C V_{H}$ induces lower $M^{*}, \widehat{M}$, and lower $L\left(M^{*}, T^{*}\right), L(\widehat{M}, \widehat{T})$, $M^{*} T^{*}$ and $\widehat{M} \widehat{T}$. Therefore, both models - true and approximate - rely less on inspections and move to preventive maintenance. As a consequence, the difference between the policies reduces, and so does the cost difference $\Delta g$.

Overall, policy $(\widehat{M}, \widehat{T})$ yields $19 \%$ higher costs, on average, compared policy $\left(M^{*}, T^{*}\right)$, which may increase to $68 \%$, dependent on the factor choice. Such a relative cost difference $\Delta g$ corresponds to large absolute cost differences, and might therefore substantially affect practical maintenance decision making. However, the obtained numerical results depend on our testbed and thus are not directly generalizable to other parameter settings. Yet, it gives an indication between the difference between both models that might occur.

\section{Extension: a reliability constraint}

In this section, we extend our model by adding a reliability constraint. The inclusion of such a reliability constraint is motivated by a large Maintenance Repair and Overhaul (MRO) organization in the Netherlands, NedTrain. NedTrain is the MRO organization of the Dutch Railways, and 
performs all inspections, maintenance and refurbishment of the rolling stock. NedTrain, is enforced by Dutch law to explicitly include reliability measures in their maintenance analysis. Such reliability aspects can be included in the cost expression, but this approach may be rather controversial, e.g., transforming human injuries into monetary terms (Aven and Castro, 2009). Furthermore, the required reliability measures imposed on NedTrain are typically given in terms of a maximum number of failures per time unit. Therefore, we propose to extend our model by including a reliability constraint. We comply to the terminology of the reliability constraint present at NedTrain. Hence, we consider a maximum average number of failures per time unit over an infinite time horizon $R_{\max }$. Similarly, we let $R(M, T)$ correspond to the average number of failures per time unit under a maintenance policy $(M, T)$, over an infinite time horizon. The reliability $R(M, T)$ is obtained similar to $g(M, T)$, i.e.,

$$
R(M, T)=\frac{F(M, T)}{L(M, T)}
$$

where $F(M, T)$ denotes the expected number of failures in a renewal cycle and $L(M, T)$ as before, see Equation (2). We derive the expression for $R(M, T)$ by using the event paths from Section 3.1. The expected number of failures in a renewal cycle $F(M, T)$ corresponds to the probability of corrective maintenance in a renewal cycle. By summing over all possible values of $i$ and $j$ for event paths of types 3 and 4 , we find

$$
F(M, T)=\sum_{i=1}^{M} \pi_{3, i}+\sum_{i=1}^{M-1} \sum_{j=i}^{M-1} \pi_{4, i, j}
$$

We obtain the following reliability constrained model with non-constant probabilities of false positives and false negatives:

$$
\begin{array}{ll}
\min _{M, T} & g(M, T) \\
\text { s.t. } & R(M, T) \leq R_{\max } \\
& T>0, M \in \mathbb{N} .
\end{array}
$$

We remark that one needs to modify the optimization procedure from Section 3.2. At each enumerated value for the inspection interval length $T_{M}$, we check whether the reliability constraint is satisfied. 


\section{Conclusion}

We considered a single-component system, which is periodically inspected and subject to imperfect inspections. We presented an exact cost evaluation of a maintenance policy - characterized by periodic inspections and preventive system maintenance - for a single-component DTM including non-constant probabilities of false positives and false negatives. Furthermore, we proposed a method that allows us to compare our true model, with non-constant probabilities of imperfect inspections, to the approximate model, that considers constant probabilities of imperfect inspections. In our numerical study, we illustrate that using the simpler model with constant probabilities of imperfect inspections can yield maintenance policies that are on average $19 \%$ more expensive than the optimal maintenance policies derived from a model with non-constant probabilities of imperfect inspections. The maximum cost difference that we found in our computational study was $68 \%$. We would like to emphasize that these numbers depend on our parameter values used.

The model described in this paper is computationally demanding for evaluation. The enumerative optimization procedure proposed in this research, can therefore become computationally prohibitive. Hence, for further research, we suggest to explore some structural properties in the maintenance policy solutions in order to cut off a part of the search space. Additionally, it would be interesting to explore other functions for the probability of false positives and false negatives in further research. Finally, one may consider to deviate from a fixed inspection interval length and study non-periodic inspections.

\section{Acknowledgements}

The authors thank the referees for their valuable comments that improved this paper. The authors would also like to express their gratitude to NedTrain for their cooperation in this project. Furthermore, we gratefully acknowledge the support of the Netherlands Foundation for Scientific Research; the Chinese Ministry of Science and Technology (Grant No. 2016YFF0203801); the Na-

tional Center for Mathematics and Interdisciplinary Sciences, Chinese Academy of Sciences; the one-hundred plan (class C) of the Chinese Academy of Sciences (No. Y629141CC1-4199900).

\section{References}

Aven, T., Castro, I. T., 2009. A delay-time model with safety constraint. Reliability Engineering \& System Safety 94 (2), 261-267. 
Berens, A. P., 1989. NDE Reliability data analysis. In: Metals Handbook, 9th Edition. Vol. 17. ASM International, pp. 689-701.

Berrade, M. D., Scarf, P. A., Cavalcante, C. A. V., Dwight, R. A., 2013. Imperfect inspection and replacement of a system with a defective state: A cost and reliability analysis. Reliability Engineering \& System Safety 120, 80-87.

Christer, A. H., 1999. Developments in delay time analysis for modelling plant maintenance. Journal of the Operational Research Society, 1120-1137.

Forsyth, D. S., Fahr, A., 1998. An evaluation of probability of detection statistics. In: Conference proceedings: Airframe Inspection Reliability under Field/Depot Conditions, RTO-MP-10, AC/323 [AVT] TP/2, Bruxelles.

Georgiou, G. A., 2006. Probability of detection (pod) curves: derivation, applications and limitations. Jacobi Consulting Limited Health and Safety Executive Research Report 454.

Jardine, A. K., Tsang, A. H., 2013. Maintenance, replacement, and reliability: theory and applications. CRC press.

Kahneman, D., 2003. Maps of bounded rationality: Psychology for behavioral economics. American Economic Review, 1449-1475.

Kallen, M.-J., van Noortwijk, J. M., 2005. Optimal maintenance decisions under imperfect inspection. Reliability Engineering \& System Safety 90 (2), 177-185.

Le, M. D., Tan, C. M., 2013. Optimal maintenance strategy of deteriorating system under imperfect maintenance and inspection using mixed inspection scheduling. Reliability Engineering \& System Safety 113, 21-29.

Mobley, R. K., 2002. An introduction to predictive maintenance. Butterworth-Heinemann.

Newby, M., Dagg, R., 2004. Optimal inspection and perfect repair. IMA Journal of Management Mathematics 15 (2), 175-192.

Nichols, J. M., Trickey, S. T., Seaver, M., Motley, S. R., 2008. Using ROC curves to assess the efficacy of several detectors of damage-induced nonlinearities in a bolted composite structure. Mechanical Systems and Signal Processing 22 (7), 1610-1622. 
Okumura, S., Jardine, A. K. S., Yamashina, H., 1996. An inspection policy for a deteriorating singleunit system characterized by a delay-time model. International Journal of Production Research $34(9), 2441-2460$.

Ross, S. M., 1983. Stochastic Processes. Wiley, New York.

Scarf, P. A., Cavalcante, C. A. V., Dwight, R. A., Gordon, P., 2009. An age-based inspection and replacement policy for heterogeneous components. IEEE Transactions on Reliability 58 (4), 641-648.

van Oosterom, C. D., Elwany, A. H., Çelebi, D., van Houtum, G. J., 2014. Optimal policies for a delay time model with postponed replacement. European Journal of Operational Research $232(1), 186-197$.

Wang, W., 2010. Modelling planned maintenance with non-homogeneous defect arrivals and variable probability of defect identification. Maintenance and Reliability 46 (2), 73-77.

Wang, W., 2012. An overview of the recent advances in delay-time-based maintenance modelling. Reliability Engineering \& System Safety 106, 165-178.

Wang, W., 2013. Models of inspection, routine service, and replacement for a serviceable onecomponent system. Reliability Engineering \& System Safety 116, 57-63.

Welte, T. M., 2009. Using state diagrams for modeling maintenance of deteriorating systems. IEEE Transactions on Power Systems 24 (1), 58-66.

Whitmore, G., 1995. Estimating degradation by a wiener diffusion process subject to measurement error. Lifetime Data Analysis 1 (3), 307-319.

Wickens, C. D., 1992. Engineering psychology and human performance. HarperCollins Publishers. 